\title{
A Study of the Relation between Distribution of Grain Diameters and Flow Stress in Mild Steel*
}

\author{
By Hiroaki MASUI** and Hiroshi TAKECHI**
}

\section{Synopsis}

Effects of grain diameter distribution on the flow stress of mild steel were studied by means of comparison between the "apparent" that is computed on the basis of the apparent average grain diameter in the usual manner and the "true" flow stress calculated taking the distribution of grain diameter into account. Three types of distribution, i.e., the Gaussian, the Poisson and the uniform, and two deformation models, i.e., the constant strain model and the constant stress model, were considered.

The following results were obtained:

(1) The stress calculated by the constant strain model and that calculated by the constant stress model agreed well for all the three types of grain diameter distribution.

(2) In Gaussian distribution $\left(f(x)=(1 / \sqrt{2} \pi S) \exp \left\{-(x-\mu)^{2} /\right.\right.$ $\left.2 S^{2}\right\}, x$ : grain diameter $\left.(\mathrm{mm})\right)$, the difference between the apparent and the true increased with $S$.

(3) In the uniform distribution $(f(x)=1 / 2 P)$, the difference increased with $P$.

(4) In Poisson distribution $\left(f(x)=\exp (-R) \cdot R^{x} / X !, X=100 x\right)$, the difference decreased with $R$, gradually approaching that for the Gaussian distribution.

(5) The fundamental formula for computation of the flow stress deduced in the present study was shown to correspond to that theoretical arrived at by Conrad, et al.

(6) The difference could be appreciable if the distribution was Poisson, but when Gaussian or uniform, it might be neglected except in cases where the flow stress was to be computed with an accuracy of the order of $10^{-1}$ $\mathrm{kg} / \mathrm{mm}^{2}$.

\section{Introduction}

One of the metallurgical factors essential for the flow stress of a mild steel is the grain diameter of the ferrite, ${ }^{1-5}$ ) and the two are known to be related by Hall-Petch's equation,

$$
\sigma=\sigma_{i}+k d^{-1 / 2}
$$

where, $\quad \sigma:$ the flow stress

$$
d: \text { the average grain diameter }
$$

$\sigma_{i}$ and $k$ : constants.

Now, to obtain the average grain diameter, we usually count the number of grains in a certain given area on a photomicrograph taken of a cross section of the specimen. But this average grain diameter is only an "apparent" average, because it is obtained without taking the volumetric true distribution of grain diameter into consideration.

Even if we could know the "true" volumetric average grain diameter, however, question would still remain as to how one should calculate the flow stress on that "true" average grain diameter. This question cannot be ignored in case of the so-called mixed grain size as sometimes encountered in mild steels where the grains vary with a certain type of diameter distribution.

For this purpose, the authors have calculated assuming appropriate mechanics models the differences between the flow stresses to be computed by the HallPetch relation on the basis of the apparent grain diameter in an usual procedure (the apparent flow stress) and those to be computed similarly but on the basis of variable grain diameters with certain volumetric distribution.

\section{Experimental Procedures}

Table 1 shows the chemical composition of the low carbon steel sheet used in the present study. The slabs were heated for $1 \mathrm{hr}$ at $1100^{\circ}, 1200^{\circ}, 1300^{\circ}$ and $1350^{\circ} \mathrm{C}$, then hot-rolled to $2.7 \mathrm{~mm}$ with a finishing temperature of $900^{\circ} \mathrm{C}$. These hot-rolled sheets were pickled in a hydrochloric acid solution and coldrolled to $0.8 \mathrm{~mm}$. Next, they were cut into specimens of $180 \mathrm{~mm}$ (rolling direction) $\times 35 \mathrm{~mm}$ (cross direction) and were annealed at $750^{\circ} \mathrm{C}$ for $4 \mathrm{hr}$ with a heating rate of $100^{\circ} \mathrm{C} / \mathrm{hr}$, then furnace cooled. They were then machined to JIS No. 5 tensile test pieces. The four heating temperatures gave rise, as expected, to four discrete levels of average grain diameters.

The tensile test was conducted at a cross head speed of $20 \mathrm{~mm} / \mathrm{min}$ with an Instron tensile tester, and the computations on the model equations were conducted with TOSBAC 3400 .

\section{Theoretical Models and Calculations}

\section{Flow Stress of Polycrystalline Mild Steel}

The true stress-true strain curves are schematically shown in Fig. 1. Although many functions between the true stress (hearafter called the "stress") $\sigma$ (kg/ $\mathrm{mm}^{2}$ ) and the true strain ("strain ") $\varepsilon$ have been advanced in the past, in a small range of the strain,

Table 1. Chemical analysis of the sample (wt\%)

\begin{tabular}{ccccccccc}
$\mathrm{C}$ & $\mathrm{Mn}$ & $\mathrm{S}$ & $\mathrm{P}$ & $\mathrm{Si}$ & $\mathrm{Cu}$ & sol. N & insol. N & $\mathrm{O}$ \\
\hline 0.021 & 0.31 & 0.019 & 0.010 & 0.012 & 0.042 & 0.0024 & 0.0002 & 0.033
\end{tabular}

* Originally published in Tetsu-to-Hagané, 58 (1972), 1107 in Japanese. English version received December 10, 1974.

** Kimitsu Works, Nippon Steel Corp., Kimitsu 299-11. 


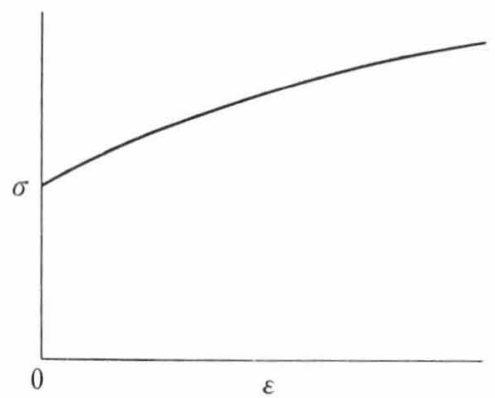

Fig. 1. Schematic representation of the stress-strain curve of mild steel

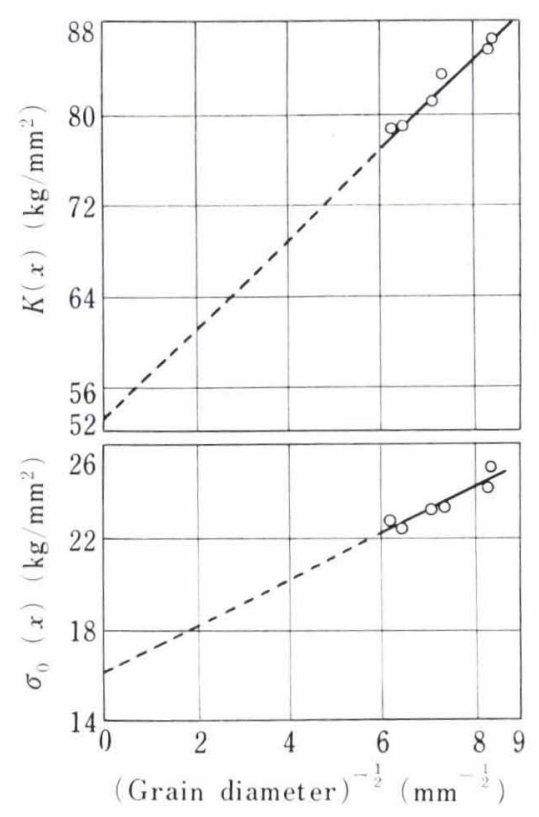

Fig. 2. Relations of $\sigma_{0}$ and $K$ to grain diameter in $\sigma=\sigma_{0}+$ $K \cdot \varepsilon$ at $\varepsilon=0.095$

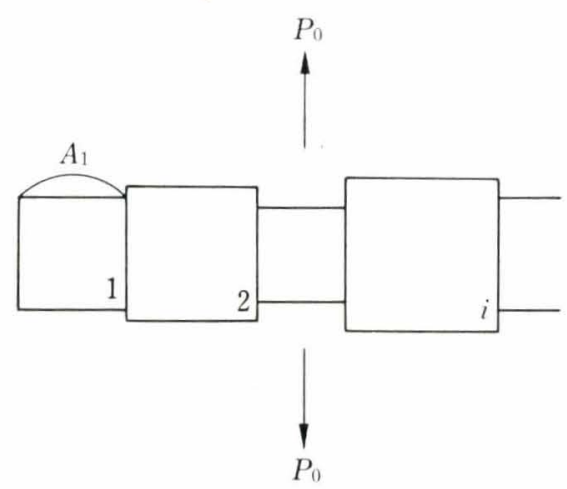

Fig. 3. Schematic representation of grains in the constant strain model

the stress may approximately be given by a linear function. Thus,

$$
\sigma=\sigma_{0}(x)+K(x) \cdot \varepsilon
$$

where, $x(\mathrm{~mm})$ is the grain diameter. Here, as shown in Fig. 2, the two terms of $\sigma_{0}(x)$ and $K(x)$ (determined at $\varepsilon=0.095$, or a nominal elongation of $10 \%$ ) were found to be related to the average grain diameter $x$ (computed according to either the JIS or the ASTM methd) through

$$
\sigma_{0}(x)=16+x^{-1 / 2}\left(\mathrm{~kg} / \mathrm{mm}^{2}\right)
$$

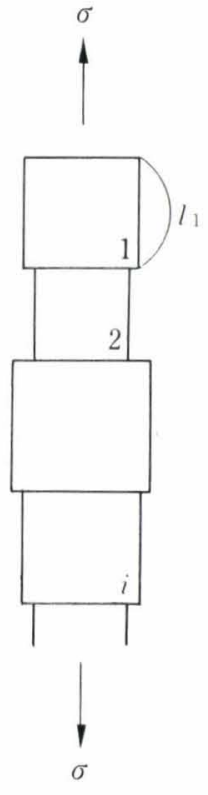

Fig. 4. Schematic representation of grains in the constant stress model

and

$$
K(x)=53+4 x^{-1 / 2}\left(\mathrm{~kg} / \mathrm{mm}^{2}\right)
$$

\section{Constant Strain Model}

As schematically shown in Fig. 3, if the stresses which are imposed upon the grains $1,2, \ldots, \mathcal{N}$ are $\sigma_{1}, \sigma_{2}, \ldots, \sigma_{N}$, and the cross sectional areas, normal to tensile axis, are $A_{1}, A_{2}, \ldots, A_{N}$, the tensile load $P_{0}$ can be expressed by Eq. (5) and the flow stress $\sigma$ by Eq. (6). Thus,

$$
\begin{aligned}
P_{0} & =\sum_{i=1}^{N} \sigma_{i} A_{i} \ldots \ldots \ldots . . \\
& =\sum_{i=1}^{N} \sigma_{i}\left(A_{i} / \sum_{i=1}^{N} A_{i}\right)
\end{aligned}
$$

Since an area fraction of a given phase is equal to its volume fraction, Eq. (6) is rewritten as

$$
\sigma\left(\equiv \sigma_{n}\right)=\int \sigma(x) \frac{x^{3} f(x)}{\left\{\int x^{3} f(x) d x\right\}} d x,
$$

where, $\sigma(x)=\sigma_{0}(x)+K(x) \varepsilon$, and $f(x)$ is a probability density function of grains having a diameter $x$.

\section{Constant Stress Model}

As schematically shown in Fig. 4, if the stress $\sigma$ which is imposed on the grains $1,2, \ldots, \mathcal{N}$ is constant, and the length and the strain of each grain along the stress axis are $l_{1}, l_{2}, \ldots, l_{N}$, and $\varepsilon_{1}, \varepsilon_{2}, \ldots, \varepsilon_{N}$, respectively, the change of total length $l, \Delta l$, is

$$
\begin{aligned}
\Delta l / l & =\sum_{i=1}^{N} \Delta l_{i} / l \\
& =\sum_{i=1}^{N}\left(l_{i} / l\right) \cdot\left(\varepsilon_{i}+\varepsilon_{i}^{2} / 2\right)
\end{aligned}
$$

For the $i$ th group of $\sigma_{0}(x)$ and $K(x)$, expressed as $\sigma_{0 i}$ and $K_{i}$ respectively, Eq. (9) holds:

$$
\sigma=\sigma_{0 i}+K_{i} \cdot \varepsilon_{i}
$$

Eliminating $\varepsilon_{i}$ from Eqs. (8) and (9), 

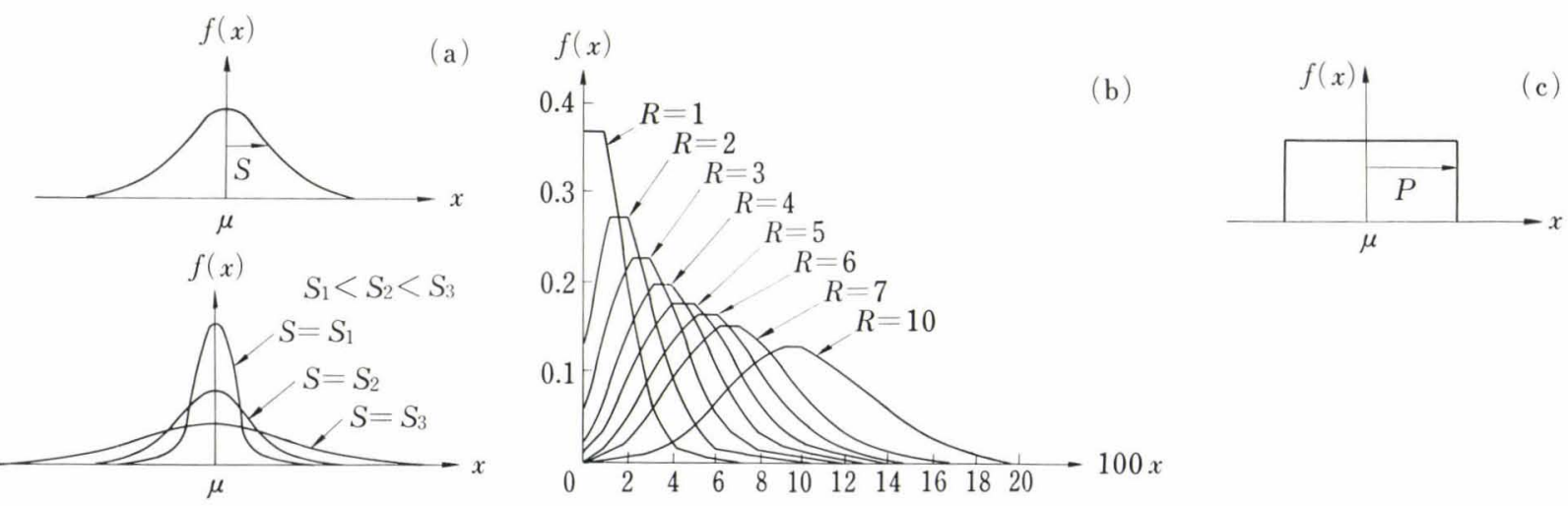

(a) Gauss: $f(x)=(1 / \sqrt{2} 2 \pi) \cdot \exp \left\{-(x-\mu)^{2} / 2 S^{2}\right\} \quad$ (b) Poisson: $f(x)=\exp (-R) \cdot R^{x} / X$ ! $(X=100 x) \quad$ (c) The uniform: $f(x)=1 / 2 P$ Fig. 5. The three probability density functions used in the calculation

$$
\frac{\Delta l}{l}=\sum_{i=1}^{N} \frac{l_{i}}{l}\left\{\begin{array}{c}
\sigma-\sigma_{0 i} \\
K_{i}
\end{array}+\frac{1}{2}\left(\frac{\sigma-\sigma_{0 i}}{K_{i}}\right)^{2}\right\}
$$

Then,

$$
\begin{aligned}
\sigma= & {\left[-\sum_{i=1}^{N} \frac{K_{i}-\sigma_{0 i}}{K_{i}^{2}} \cdot \frac{l_{i}}{l}+\left\{\left(\sum_{i=1}^{N} \frac{K_{i}-\sigma_{0 i}}{K_{i}^{2}} \cdot \frac{l_{i}}{l}\right)^{2}\right.\right.} \\
& -\left(\sum_{i=1}^{N} \frac{1}{K_{i}^{2}} \frac{l_{i}}{l}\right) \cdot\left(\sum_{i=1}^{N} \frac{\sigma_{0 i}^{2}-2 K_{i} \sigma_{0 i}}{K_{i}^{2}} \cdot \frac{l_{i}}{l}\right. \\
& \left.\left.\left.-2 \frac{\Delta l}{l}\right)\right\}^{1 / 2}\right] / \sum_{i=1}^{N} \frac{1}{K_{i}^{2}} \cdot \frac{l_{i}}{l} \quad \ldots \ldots \ldots \ldots \ldots \ldots \ldots \ldots \ldots \ldots
\end{aligned}
$$

Rewriting Eq. (11), using the probability density function of grain diameter $f(x)$,

$$
\sigma\left(\equiv \sigma_{s}\right)=\left[-\Gamma_{2}+\left\{\Gamma_{2}^{2}-\Gamma_{1}\left(\Gamma_{3}-2 D\right)\right\}^{1 / 2}\right] / \Gamma_{1}
$$

where,

$$
\begin{aligned}
\Gamma_{1} & =\int\left\{1 /(K(x))^{2}\right\} \cdot F(x) \cdot d x \\
\Gamma_{2} & =\int\left\{\left(K(x)-\sigma_{0}(x)\right) /(K(x))^{2}\right\} \cdot F(x) d x \\
\Gamma_{3} & =\int\left[\left\{\left(\sigma_{0}(x)\right)^{2}-2 K(x) \sigma_{0}(x)\right\} /(K(x))^{2}\right] \cdot F(x) d x \\
D & =e^{\varepsilon}-1 \\
F(x) & =x^{3} f(x) / \int x^{3} f(x) d x
\end{aligned}
$$

\section{Probability Density Function, $f(x)$}

For the probability density function $f(x)$, the Gauss distribution, the Poisson distribution and the uniform distribution were selected as shown in Fig. 5.

Gauss distribution:

$$
f(x)=\{1 /(\sqrt{ } 2 \pi S)\} \cdot \exp \left\{-(x-\mu)^{2} /\left(2 S^{2}\right)\right\} .
$$

Poisson distribution:

$$
f(x)=\exp (-R) \cdot R^{x} / X !, \quad(X=100 x)
$$

Uniform distribution:

$$
f(x)=\left\{\begin{array}{l}
1 /(2 P), \quad \mu-P \leq x \leq \mu+P \\
0, \quad x<\mu-P, \quad x>\mu+P
\end{array}\right.
$$

\section{Results of Galculation}

In the two models of constant strain and constant stress, flow stresses were calculated for the Gauss distribution, the uniform distribution and the Poisson

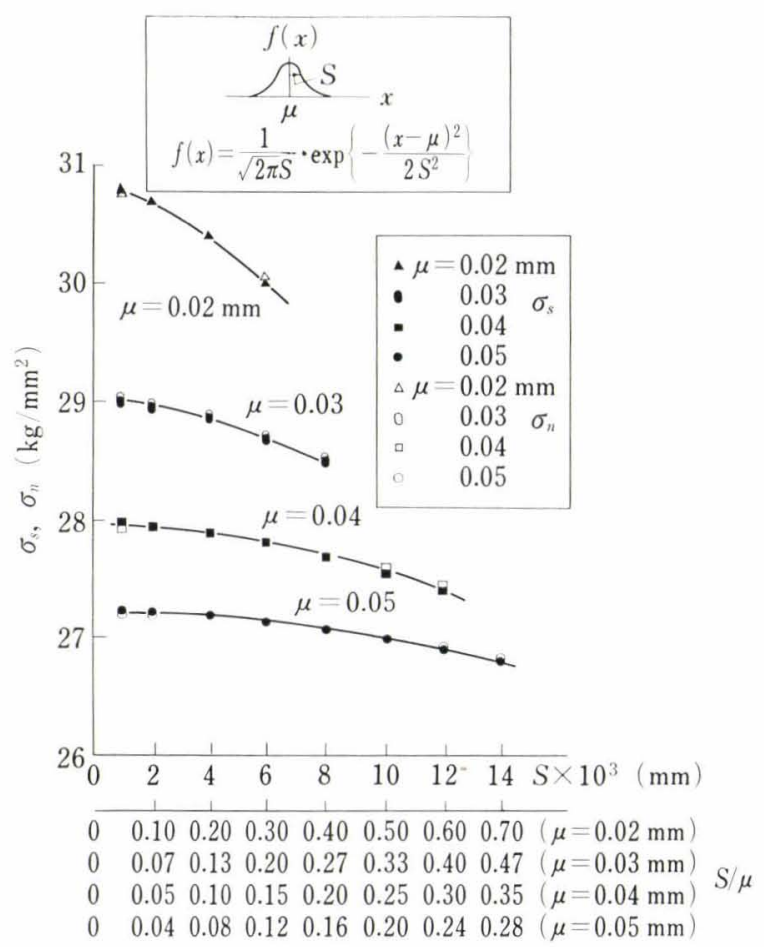

Fig. 6. Changes in the flow stress, $\sigma_{s}$ or $\sigma_{n}$, for Gaussian grain diameter distribution with $S$ or $S / \mu$ as the parameter

distribution by substituting Eqs. (3), (4), (13), and (15) into Eqs. (7) and (12).

The results are shown in Fig. 6 (the Gauss distribution), in Fig. 7 (the uniform distribution) and in Fig. 8 (the Poisson distribution), respectively. In the figure, the ways the flow stress changes in accordance with the changes in $S$ (or $S / \mu$ ) for Gaussian distribution, $P$ (or $P / \mu$ ) for uniform distribution, and $R$ for Poisson distribution are seen. It is to be noted, further, that the two flow stresses, one corresponding to the constant strain model and the other to the constant stress model, always agree each other well.

Now, for the deformation of polycrystals, the most important condition is that every grain should satisfy both the displacement continuity condition and the traction continuity condition at the same time. In other words, a flow stress which satisfies both conditions at the same time may be considered the real 


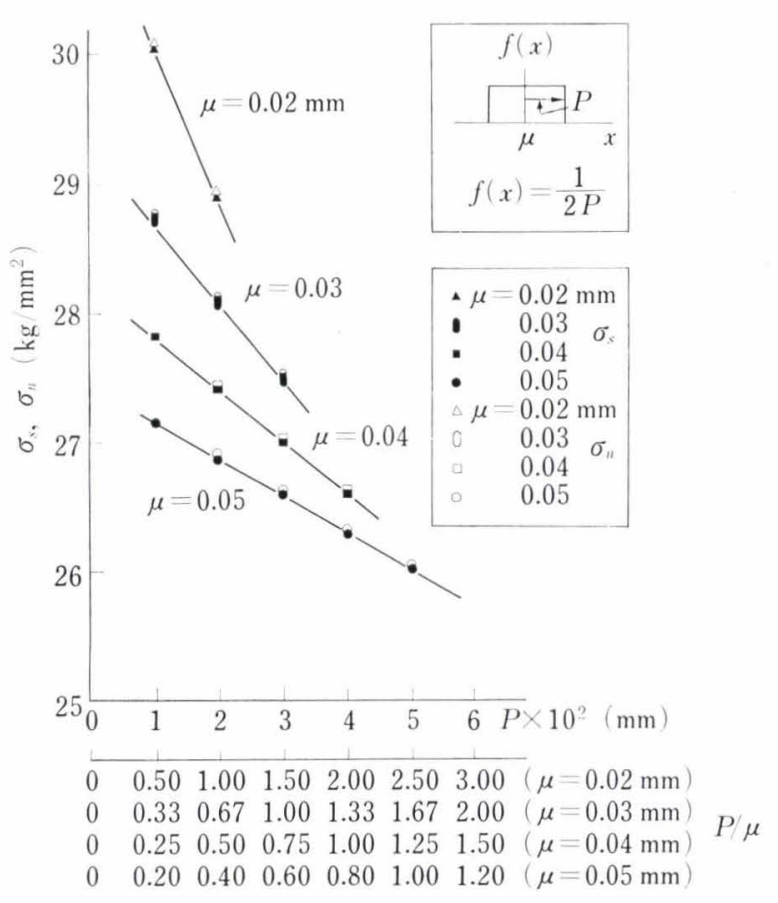

Fig. 7. Changes in the flow stress, $\sigma_{s}$ or $\sigma_{n}$, for the uniform grain diameter distribution with $P$ or $P / \mu$ as the parameter

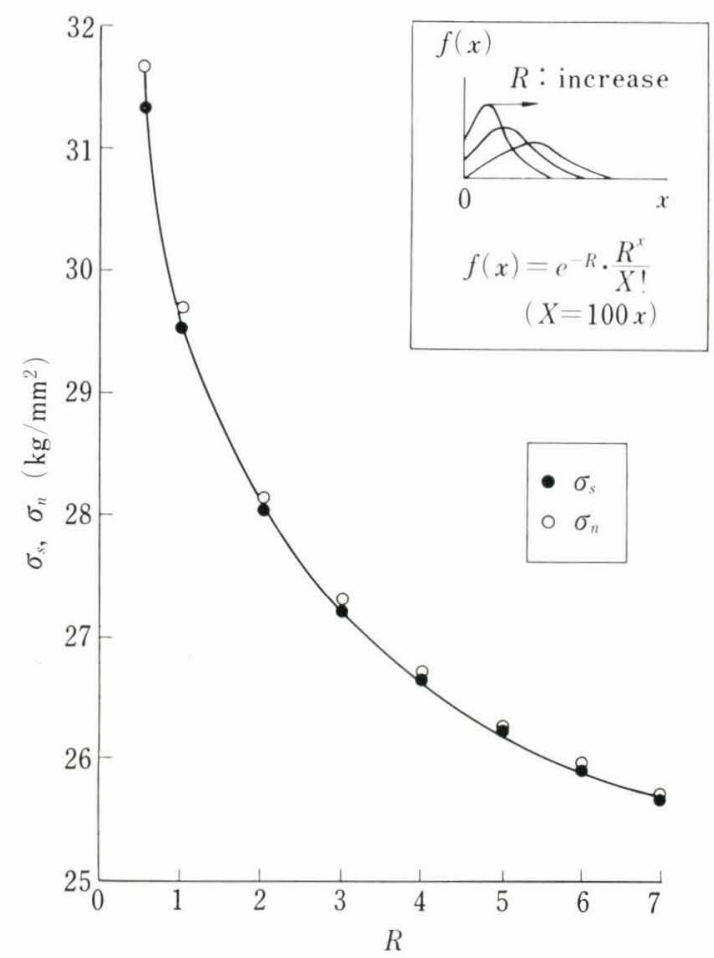

Fig. 8. Changes in the flow stress $\sigma_{s}$ or $\sigma_{n}$, for Poisson grain diameter distribution with $R$ as the parameter

true stress. Therefore, with regard to the flow stress calculated by the constant strain model, $\sigma_{n}$ (Eq. (7)), and the one by the constant stress model, $\sigma_{s}$ (Eq. (12)), the arithmetical mean of the two will be very near to the real flow stress of that polycrystal, so that such stress may be named the true flow stress $\sigma_{t r}$. Therefore,

$$
\sigma_{t r}=\left(\sigma_{n}+\sigma_{s}\right) / 2
$$

2. Structure in Any Cross Section of a Polycrystal and the Apparent Average Grain Diameter

The common method we obtain the apparent grain diameter is to take a photomicrograph of a cross section of the polycrystal concerned and to count the number of grains in a certain given area of the photograph.

Now, we will see what the apparent average grain diameter thus determined will be in different types of distribution. Here, we assume the grains to be globular.

\section{The Apparent Average Grain Diameter in the Globular}

Model

The probability that the radius of a circle that is formed in a globe (radius $R_{0}$ ) when cut by a plane at any position will be within $r$ and $r+d r$ is,

$$
P(r) d r=\frac{1}{R_{0}} \cdot \frac{r}{\sqrt{R_{0}^{2}-r^{2}}} d r
$$

The average area $\pi r^{2}$ for circles appearing in a plane that cuts a volume completely filled with globes of radius $R_{0}$ at any position is, therefore,

$$
\begin{aligned}
\pi r^{2} & =\int_{0}^{R_{0}} \pi r^{2} P(r) d r \\
& =(2 / 3) \pi R_{0}^{2} \quad \ldots
\end{aligned}
$$

Accordingly, if the total number of circles observed in a certain area $A_{0}$ of the cutting plane is $\mathcal{N}_{A_{0}}$,

$$
\mathcal{N}_{A_{0}}=A_{0} /\left\{(2 / 3) \pi R_{0}^{2}\right\}
$$

When globes of various diameters $x_{1}, x_{2}, \ldots, x_{M}$ are filling in the volume,

$$
\begin{gathered}
\mathcal{N}_{A_{1}}=A_{1} /\left\{(2 / 3) \pi \cdot\left(x_{1} / 2\right)^{2}\right\}=A_{1} /\left\{(1 / 6) \pi x_{1}^{2}\right\} \\
\mathcal{N}_{A_{2}}=A_{2} /\left\{(1 / 6) \pi x_{2}^{2}\right\} \\
\vdots \\
\mathcal{N}_{A_{M}}=A_{M} /\left\{(1 / 6) \pi x_{M}^{2}\right\} \quad \ldots \ldots \ldots \ldots \ldots \ldots \ldots \ldots \ldots \ldots \ldots \ldots \ldots \ldots
\end{gathered}
$$

and, if the total area is $A_{T}$ and the total number of gloves observed in $A_{T}$ is $\mathcal{N}_{M}$,

$$
\begin{aligned}
& A_{T}=A_{1}+A_{2}+\ldots+A_{M} \\
& \mathcal{N}_{M}=\mathcal{N}_{A_{1}}+\mathcal{N}_{A_{2}}+\ldots+\mathcal{N}_{A_{M}}
\end{aligned}
$$

It follows then that, if the total volume is filled with globes of a constant diameter $\bar{x}_{a p}$,

$$
(1 / 6) \pi \bar{x}_{a p}^{2}=A_{T} / \mathcal{N}_{M}
$$

and this $\bar{x}_{a p}$ is nothing but the so-called apparent average grain diameter according to JIS or ASTM. Substituting Eqs. (20) to (22) into Eq. (23),

$$
\begin{aligned}
\frac{1}{6} \pi \bar{x}_{a, p} & =1 /\left[\frac{A_{1}}{A_{T}} \cdot \frac{1}{(1 / 6) \pi x_{1}^{2}}+\ldots+\frac{A_{M}}{A_{T} \cdot \frac{1}{(1 / 6) \pi x_{M}^{2}}}\right] \\
& =\frac{1}{6} \pi /\left[\frac{x_{1} f\left(x_{1}\right)}{\sum_{i=1}^{M} x_{i}^{3} f\left(x_{i}\right)}+\ldots+\frac{x_{M} f\left(x_{M}\right)}{\sum_{i=1}^{M} x_{i}^{3} f\left(x_{i}\right)}\right] \ldots
\end{aligned}
$$

From this relation, we finally obtain, 


$$
\bar{x}_{a p}=\left[\begin{array}{l}
\int x f(x) d x \\
\int x^{3} f(x) d x
\end{array}\right]^{-1 / 2}
$$

where, $f(x)$ is the probability density function for globes of diameter $x$.

\section{Distribution of Diameter for Circles Appearing in a Cutting Plane}

From photomicrographs of electrolytic iron samples A and B, approximate diameters of grains were measured and their distributions determined. As shown in Fig. 9, in the sample A, the distribution seems like Gaussian, whereas in B, it is more like Poisson.

The distributions shown in Fig. 9 pertain, however, merely to the two-dimensional approximate grain diameters. Therefore, a relation between the distribution of two-dimensional grains and the true distribution of grains in a volume should be sought. This may be done in the present globe model by calculating by Eq. (17) the appearance probabilities of different radius circles when the position of the cutting plane is varied as shown in Fig. 10. Result of the calculation is also shown in Fig. 10(b).

It will be seen that, if the globe radius is $R_{0}$, the probability that a circle having a radius of $0.8 R_{0}-R_{0}$ will appear in the plane of cut is about $60 \%$. This means that the observed distributions of the two-

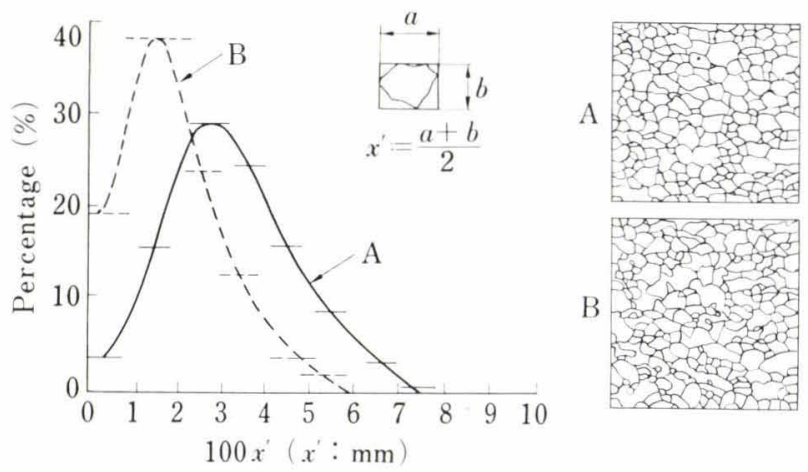

Fig. 9. Distributions of the approximate grain diameters determined on the cross section of electrolytic irons A and $\mathrm{B}$

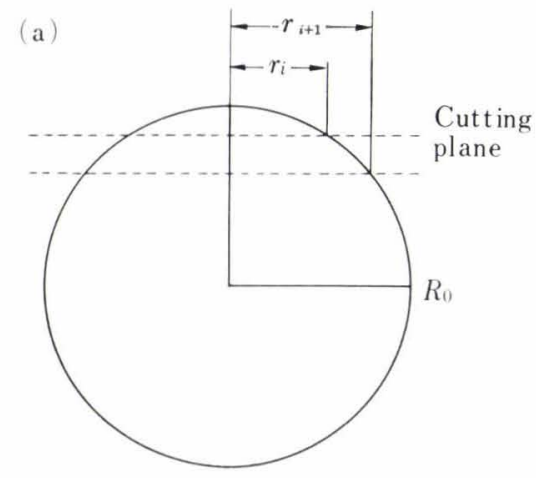

dimensional approximate grain diameters determined for the two electrolytic iron samples corresponds, roughly speaking, well to the true distribution of threedimensinal grain diameters.

\section{Difference between the Apparent Flow Stress and the True Flow Stress}

Strictly speaking, the apparent average grain diameter $\bar{x}_{a p}$ may be directly related to the flow stress only in a polycrystal whose grain diameter is constant. In this case, the apparent flow stress coincides with the true flow stress. Thus,

$$
\bar{\sigma}_{a p}=\sigma_{0}\left(\bar{x}_{a p}\right)+K\left(\bar{x}_{a p}\right) \cdot \varepsilon
$$

In real polycrystals, however, the grain diameter is variant, so that the $\bar{\sigma}_{a p}$ obtained by Eq. (26) can only be the apparent flow stress.

Therefore, for cases where the grain diameter $(x)$ is distributed with different probability density functions $f(x)$ discussed earlier, the differences between the true flow stresses $\sigma_{t r}$ and the apparent flow stresses $\bar{\sigma}_{a p}$ were calculated at $\varepsilon=0.095$ by using Eqs. (3), (4), (7), (12), (16), (25) and (26).

Figure 11 shows $\left(\bar{\sigma}_{a p}-\sigma_{n}\right)$ and $\left(\bar{\sigma}_{a p}-\sigma_{s}\right)$ with respect to both $S$ and $S / \mu$ in Eq. (13) of Gaussian distribution. As was mentioned previously, since $\sigma_{n}$ and $\sigma_{s}$ are nearly equal for changes both in $S$ and in $S / \mu$, the $\left(\bar{\sigma}_{a p}-\sigma_{n}\right)$ naturally coincides well with $\left(\bar{\sigma}_{a p}-\sigma_{s}\right)$. Therefore, as discussed in III.1. (Eq. (16)), the $\left(\bar{\sigma}_{a p}-\sigma_{t r}\right)$ may be taken for either. This is shown in Fig. 12. Similarly, the cases of the uniform distribution and of Poisson distribution are shown in Figs. 13 and 14, respectively.

It will be observed that $\left(\bar{\sigma}_{a p}-\sigma_{\iota r}\right)$ is small when the distribution is Gaussian or uniform, whereas it is relatively large when Poisson. This is emphasized in Figs. 15 and 16 by comparing $\left(\bar{\sigma}_{a p}-\sigma_{t r}\right)$ and $\left(\bar{\sigma}_{a p}\right.$ $\left.-\sigma_{t r}\right) / \sigma_{t r}$ between Gaussian distribution and Poisson distribution.

Now, it can be proved mathematically that the probability density function of Poisson distribution becomes to resemble that of Gaussian distribution for large $R$ 's. ${ }^{6)}$ This theorem applies to the cases at hand as follows: in Fig. 15, the probability density function of a Gaussian distribution for a $\mu$ of 0.05 ( $\mathrm{mm}$ ) should

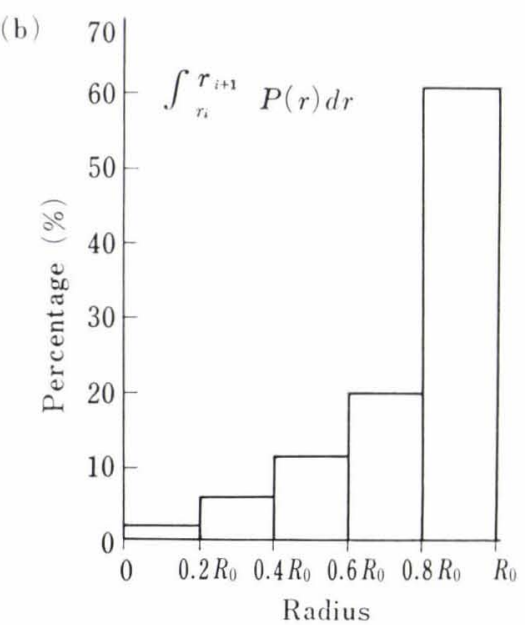

Fig. 10. Distribution of the radii appearing in planes cutting spherical grain of radius $R_{0}$ 


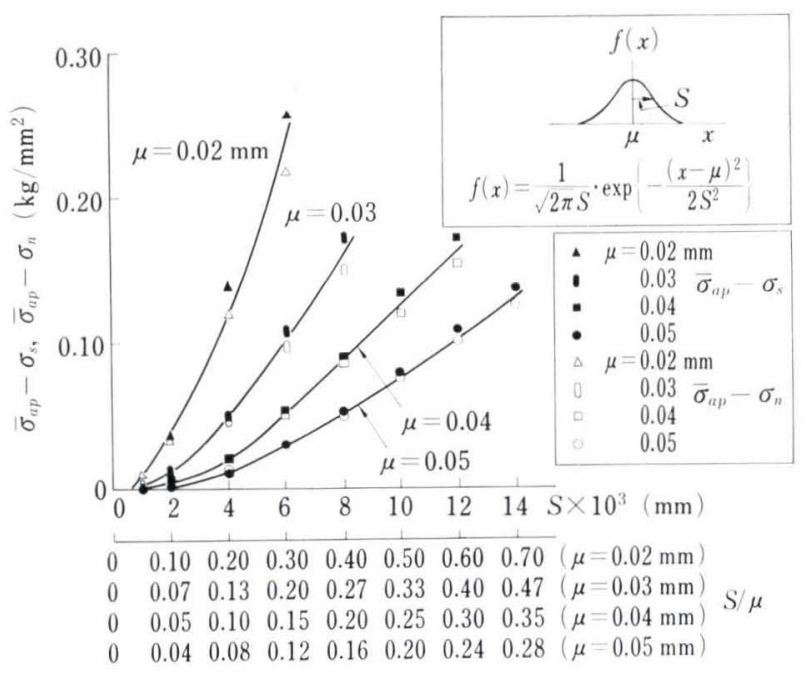

Fig. 11. Differences between $\bar{\sigma}_{a p}$ and $\sigma_{s}$ or $\sigma_{n}$ for Gaussian distribution with $S$ or $S / \mu$ as the parameter

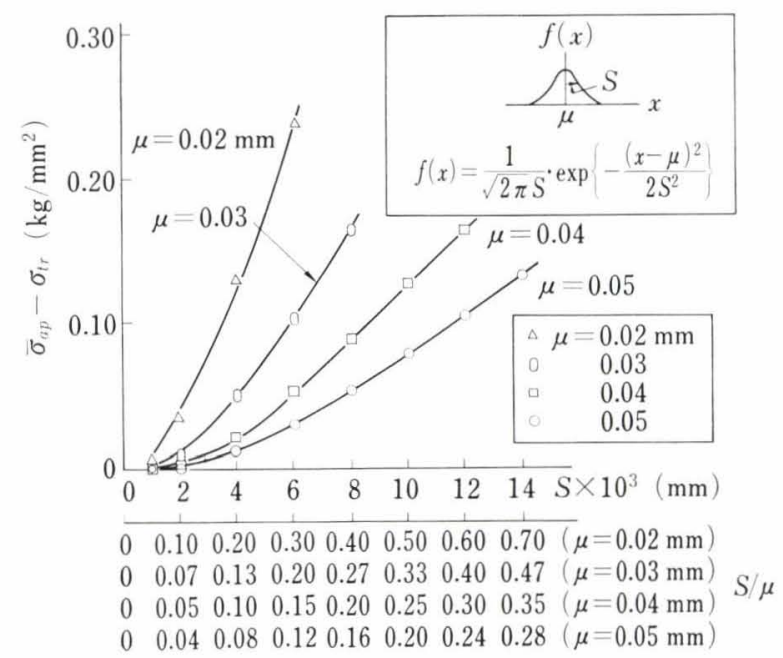

Fig. 12. Differences between $\bar{\sigma}_{a p}$ and $\sigma_{t r}$ for Gaussian distribution with $S$ or $S / \mu$ as the parameter

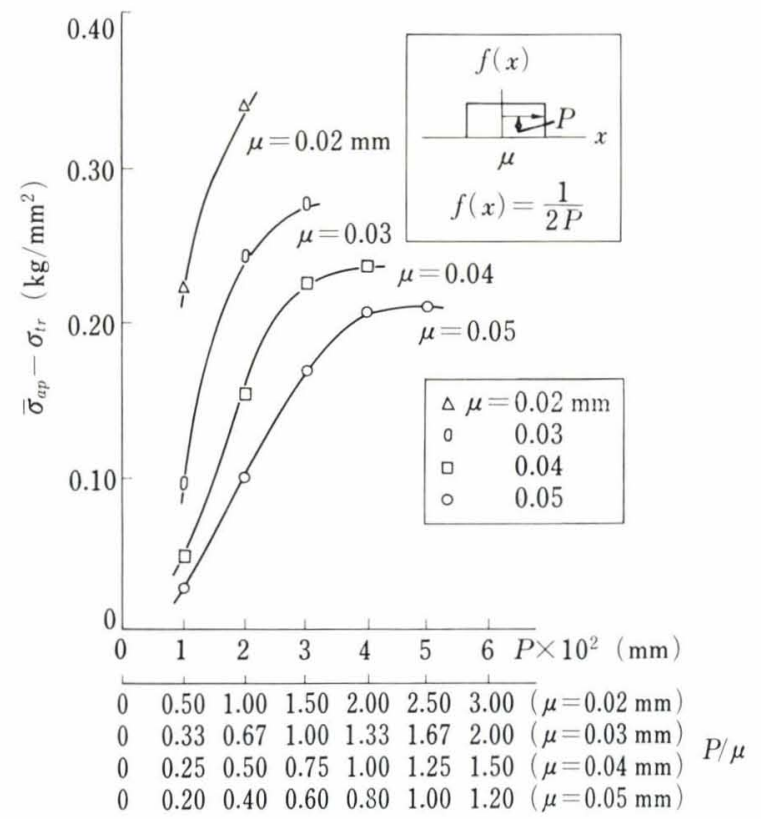

Fig. 13. Differences between $\bar{\sigma}_{a p}$ and $\sigma_{t r}$ for the uniform distribution with $P$ or $P / \mu$ as the parameter

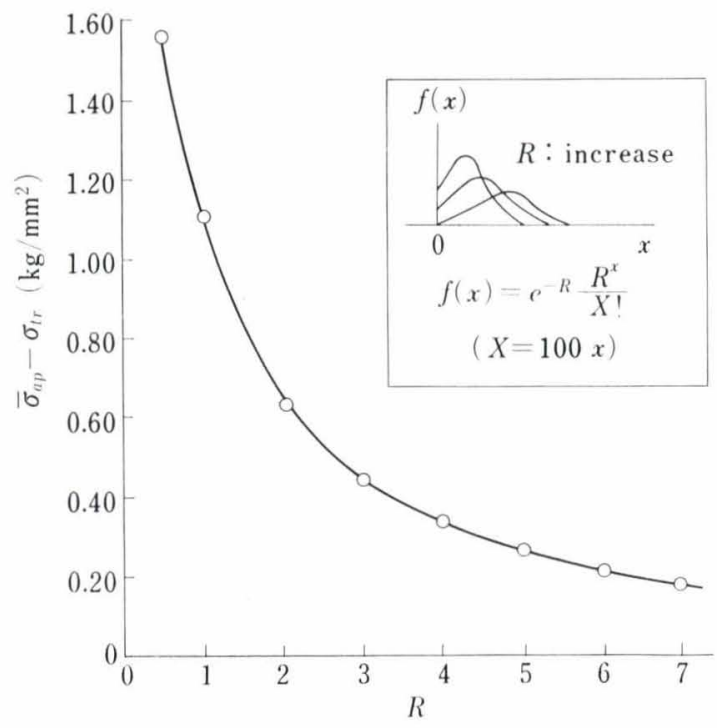

Fig. 14. Differences between $\bar{\sigma}_{a p}$ and $\sigma_{t r}$ for Poisson distribution with $R$ as the parameter

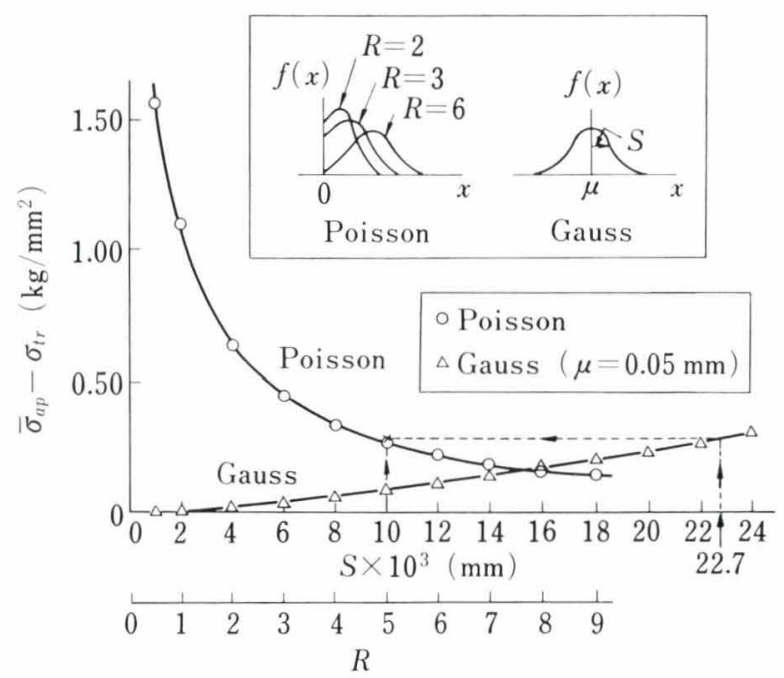

Fig. 15. Comparison of $\bar{\sigma}_{a p}-\sigma_{t r}$ in Gaussian distribution and that in Poisson distribution

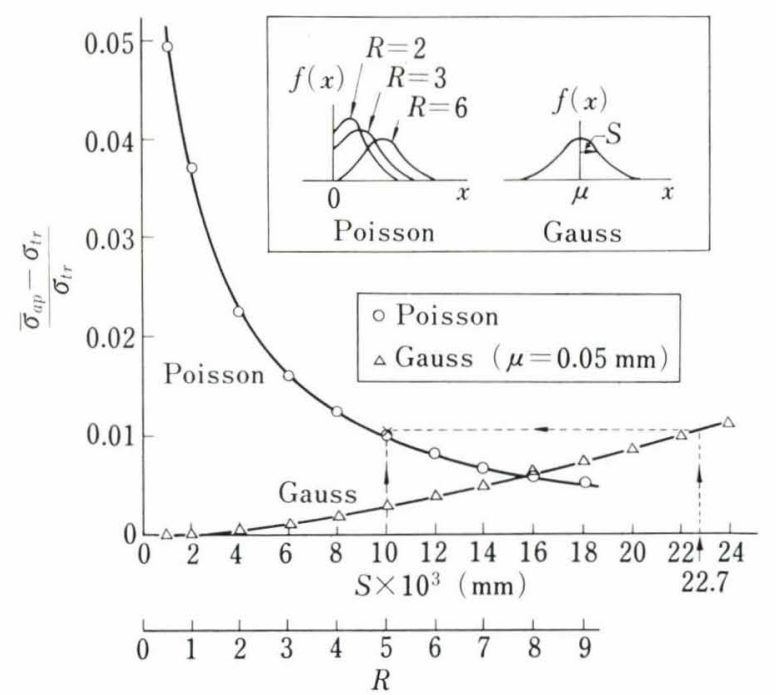

Fig. 16. Comparison of $\left(\bar{\sigma}_{a p}-\sigma_{t r}\right) / \sigma_{t r}$ in Gaussian distribution and that in Poisson distribution 
resemble very much that of Poisson distribution for an $R$ of 5 , provided a suitable value is found for $S$ in the former function, when the two $\left(\bar{\sigma}_{a p}-\sigma_{t r}\right)$ 's should agree closely each other. This value of $S$ can be calculated by equating the $1 /\left\{\sqrt{2 \pi}\left(S \times 10^{2}\right)\right\}$ term of Eq. (13) to 0.175 , which is the maximum value of Eq. (14) at $R=5$, giving an $S$ of $22.7 \times 10^{-3} \mathrm{~mm}$. Entering Fig. 15 with this value, one reads the value of $\left(\bar{\sigma}_{a p}-\sigma_{t r}\right)$ for the Gaussian distribution as marked by $\times$, which agrees remarkably well with that for $R=5$ in the Poisson distribution. The same holds true for Fig. 16.

Lastly, for comparison's sake, we introduce another kind of apparent flow stress $\bar{\sigma}$ which is defined by the "true" volumetric average grain diameter, $\bar{x}$, as follows:

$$
\begin{gathered}
\bar{x}=\int x f(x) d x \\
\bar{\sigma}=\sigma_{0}(\bar{x})+K(\bar{x}) \cdot \varepsilon
\end{gathered}
$$

Comparison between $\left(\bar{\sigma}_{a p}-\sigma_{t r}\right)$ and $\left(\bar{\sigma}-\sigma_{t r}\right)$ are shown in Fig. 17. It will be seen that, though the general trend is similar, the former is always smaller than the latter. The conclusion to be drawn is obvious.

\section{Discussion}

For the two models of constant strain (Eq. (7)) and constant stress (Eq. (12)) to uphold rigorously, a condition that each and any grain should satisfy Eqs. (2) to (4) is needed. This necessitates to introduce the work hardening as a purely metallurgical problem.

Now, Conrad, et al. ${ }^{13,14)}$ have advanced a work hardening theory which was demonstrated with niobium. We shall follow their agreement.

The flow stress (shear stress) $\tau$ may be related to the dislocation density $\rho$ in a well known relationship, ${ }^{7-12)}$

$$
\tau=\tau^{*}+\alpha \mu b \sqrt{\rho}
$$

where, $\tau^{*}:$ frictional stress

$\mu$ : shear modulus

$b$ : Burgers vector.

Since the average distance $\bar{s}$ that a dislocation glides during deformation is proportional to grain diameter, then, if grain diameter is $l$,

$$
\bar{s}=\beta l
$$

where, $\beta$ is a proportionality coefficient.

Therefore, for a shear strain $\gamma$, which is

$$
r=\rho b \bar{s},
$$

the dislocation density is given by

$$
\rho=\gamma /(b \beta l) \text {. }
$$

Substituting Eq. (32) into Eq. (29), we obtain,

$$
\tau=\tau^{*}+\alpha \mu b \sqrt{\gamma /(b \beta)} \cdot l^{-1 / 2}
$$

Now, we will modify the Eq. (33) as follows: In
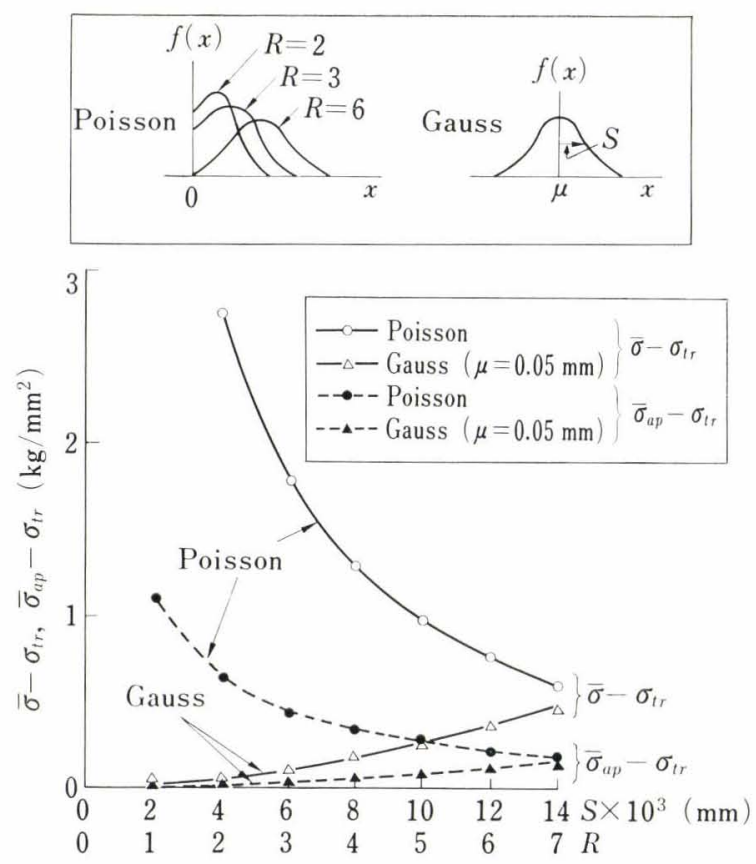

Fig. 17. Comparison of $\bar{\sigma}-\sigma_{t r}$ and $\bar{\sigma}_{a p}-\sigma_{t r}$ in Gaussian and those in Poisson distribution

terms of the tensile stress $\sigma$ and strain $\varepsilon$, Eq. (33) can be expressed approximately as

$$
\sigma=\sigma^{*}+2 \sqrt{ } 2 \alpha \mu b \sqrt{\varepsilon /(b \beta)} \cdot l^{-1 / 2}
$$

where, $\sigma^{*}=2 \tau^{*}$.

If the $\sigma-\varepsilon$ curve can be expressed approximately as a linear function for small changes in the neighborhood of $\sigma=\sigma_{0}^{\prime}\left(\right.$ at $\left.\varepsilon=\varepsilon_{0}\right)$ and $\varepsilon=\varepsilon_{0}$, and writing $2 \sqrt{2} \alpha \mu b$ / $\sqrt{b \beta} \equiv C$,

$$
\begin{aligned}
\sigma & =\sigma_{0}^{\prime}+\left.\frac{d \sigma}{d \varepsilon}\right|_{\varepsilon=\varepsilon_{0}} \cdot\left(\varepsilon-\varepsilon_{0}\right) \\
& =\sigma_{0}^{\prime}+(1 / 2) C \varepsilon_{0}^{-1 / 2} l^{-1 / 2}\left(\varepsilon-\varepsilon_{0}\right) .
\end{aligned}
$$

Substituting $\sigma_{0}^{\prime}=\sigma^{*}+C \varepsilon_{0}^{1 / 2} l^{-1 / 2}$ into Eq. (35), we obtain finally,

$$
\sigma=\sigma^{*}+(1 / 2) C \varepsilon_{0}^{1 / 2} l^{-1 / 2}+(1 / 2) C \varepsilon_{0}^{-1 / 2} l^{-1 / 2} \cdot \varepsilon \ldots
$$

It will be noticed that Eq. (36) is in the same form as Eq. (2), which together with Eqs. (3) and (4), is expressed as follows:

$$
\sigma=16+x^{-1 / 2}+\left(53+4 x^{-1 / 2}\right) \cdot \varepsilon
$$

Then, numerical comparison between Eqs. (36) and (37) is possible. Namely, in Eq. (36), the two coefficients may be evaluated by substituting $\alpha=0.4$ $\left(\mathrm{Keh}^{11)}\right), \quad \beta=0.5, \mu=7.9 \times 10^{3} \mathrm{~kg} / \mathrm{mm}^{2}, \quad b=2.48 \times 10^{-7}$ $\mathrm{mm}$, and $\varepsilon_{0}=0.095$. The results are

$$
(1 / 2) C \varepsilon_{0}^{1 / 2}=0.97 \mathrm{~kg} / \mathrm{mm}^{3 / 2}
$$

and

$$
(1 / 2) C \varepsilon_{0}^{-1 / 2}=10.2 \mathrm{~kg} / \mathrm{mm}^{3 / 2} .
$$

These values are compared with the counterparts of Eq. (37) in Table 2. It will be seen in Table 2 that, in Eqs. (36) and (37), the numerical values for 
Table 2. Comparison of coefficients in Eqs. (36) and (37)

\begin{tabular}{l|c|c|c} 
Equation & $\begin{array}{c}\text { Coefficient of } l^{-1 / 2} \\
\text { (or } x^{-1 / 2} \text { ) in the } \\
\text { term which does } \\
\text { not include } \varepsilon\end{array}$ & $\begin{array}{c}\text { Coefficient of } l^{-1 / 2} \\
\text { (or } x^{-1 / 2} \text { in the } \\
\text { term which } \\
\text { includes } \varepsilon\end{array}$ & $\begin{array}{c}\text { Coefficient } \\
\text { of } \varepsilon\end{array}$ \\
\hline Eq. (36) & 0.97 & 10.2 & $10.2 l^{-1 / 2}$ \\
Eq. (37) & 1 & 4 & $53+4 x^{-1 / 2}$ \\
\hline
\end{tabular}

coefficients of $l^{-\mathbf{1} / \mathbf{2}}\left(\right.$ or $\left.x^{-1 / 2}\right)$ in the term which does not include $\varepsilon$ agree well each other, whereas the agreement between the numerical values for coefficients of $l^{-1 / 2}\left(x^{-1 / 2}\right)$ in the term which includes $\varepsilon$ is poor. With respect to the whole term, however, that is, taking $l^{-1 / 2}\left(\right.$ or $\left.x^{-1 / 2}\right)=8\left(\mathrm{~mm}^{-1 / 2}\right)$, for example,

$$
10.2 l^{-1 / 2}=81.6 \mathrm{~kg} / \mathrm{mm}^{2},
$$

and

$$
53+4 x^{-1 / 2}=85.0 \mathrm{~kg} / \mathrm{mm}^{2} .
$$

The difference between these values is only about $4 \%$, which is not so large as compared with the experimental error. Therefore, we conclude that no definite difference exists between the two equations.

The facts that Eqs. (36) and (37) agree well each other, and that Eq. (29) applies both to single crystals and to polycrystals ${ }^{8)}$ appear to attest to the rightness of the fundamental supposition that each and any grain satisfies Eq. (37).

Finally, if we are to take certain experimental results, though their physical meaning is not well understood, that $\sigma^{*}$ increases proportionally with $\varepsilon,{ }^{15,16)}$ Eq. (36) may be written in a form more similar to Eq. (37) as follows:

$$
\sigma=\sigma^{* \prime}+(1 / 2) C \varepsilon_{0}^{1 / 2} l^{-1 / 2}+\left\{k_{0}+(1 / 2) C \varepsilon_{0}^{-1 / 2} l^{-1 / 2}\right\} \cdot \varepsilon
$$

\section{Summary and Conclusion}

We usually correlate the observed flow stress with the apparent average grain diameter which we obtain by counting the number of grains in a certain area of photomicrograph taken of the sample. Strictly speaking, this method is questionable for those samples whose grains are much varied diameter-wise. Accordingly, the flow stresses for three different types of grain diameter distribution have been calculated and compared with the apparent flow stresses which are computed simply on the apparent grain diameter.

The results are as follows.

(1) In any of the three types of volumetric grain diameter distribution, the flow stress calculated by the constant strain model coincides well with that calculated by the constant stress model. Therefore, the arithmetical mean of the two stresses should be taken as the true flow stress.

(2) When the grain diameter $(x, \mathrm{~mm})$ is in the Gaussian distribution $\left(f(x)=(1 / \sqrt{ } 2 \pi S) \cdot \exp \left\{-(x-\mu)^{2} /\right.\right.$ $\left.\left.2 S^{2}\right\}\right)$, the difference between the true flow stress and the apparent flow stress increases with $S$.

(3) When the distribution is uniform $(f(x)=1 / 2 P)$, the difference increases with $P$.

(4) When the distribution is Poisson $(f(x)=$ $\left.\exp (-R) \cdot R^{x} / X !, \quad X=100 x\right)$, the difference is comparatively large for small $R$ 's, but with increase of $R$, the difference becomes progressively smaller as the Poisson distribution approaches the Gaussian.

(5) The fundamental relation for the flow stress $\left(\sigma=\sigma_{0}(x)+K(x) \cdot \varepsilon\right.$, where, $\sigma_{0}(x)=16+x^{-1 / 2}\left(\mathrm{~kg} / \mathrm{mm}^{2}\right)$, $\left.K(x)=53+4 x^{-1 / 2}\left(\mathrm{~kg} / \mathrm{mm}^{2}\right)\right)$, which is used in obtaining the flow stress with relation to the distribution of grain diameters, corresponds to the flow stress theoretically formulated by Conrad and his associates to be acting on each grain.

(6) In summary, when the volumetric grain diameter is in the Poisson distribution, the apparent average grain diameter should not be used. When the distribution is either Gaussian or uniform, on the other hand, no great errors will result by using the apparent average grain diameter, except when discussing flow stresses of, roughly speaking, smaller than $1 \mathrm{~kg} / \mathrm{mm}^{2}$ at a precision of the order of $10^{-1} \mathrm{~kg} / \mathrm{mm}^{2}$.

\section{REFERENCES}

1) H. Masui, M. Kawaharada and H. Takechi: Tetsu-toHagané, 56 (1970), S 124.

2) W. B. Morrison: Trans. ASM, 59 (1966), 824.

3) K. Matsudo and T. Shimomura: Tetsu-to-Hagané, 56 (1970), 28.

4) W. B. Morrison and R. L. Miller: Paper to be published in Proceedings of 16 the Sagamore Conference (1969), 614.

5) T. Ichiyama, M. Koizumi, I. Yoshida, K. Watanabe and S. Nishiumi: Tetsu-to-Hagané, 55 (1969), 1229.

6) K. Narujitsu and T. Sakai: Essence of Mathematical Statistics, Baihukan Co., (1952), 41.

7) J. E. Bailey and P. B. Hirsch: Phil. Mag., 5 (1960), 485.

8) J. D. Livingston: Acta Met., 10 (1962), 229.

9) Z. B. Basinski and S. J. Basinski: Phil. Mag., 9 (1964), 51.

10) J.A. Venables: Phil. Mag., 7 (1962), 1969.

11) A.S. Keh: Direct Observation of Imperfections in Crystals, Interscience, (1962), 213.

12) D. J. Dingley and D. McLean: Acta Met., 15 (1967), 885.

13) H. Conrad, S. Feuerstein and L. Rice: Mat. Sci. Eng., 2 (1967), 157.

14) H. Conrad, S. Feuerstein and L. Rice: Trans. JIM, 9 (1968), 481.

15) Y. Ohba: Trans. Nat. Res. Inst. Metals, 9 (1967), 293.

16) J. D. Meakin and N. J. Petch: Symposium on the Role of Substructure in the Mechanical Behavior of Metals, (1963), 243. 